\title{
IMS Instrumentation I: Isolated data acquisition for ion mobility spectrometers with grounded ion sources
}

\author{
Martin Lippmann $^{1}$ (D) $\cdot$ Ansgar T. Kirk $^{1}$ (D) $\cdot$ Moritz Hitzemann $^{1}$ (D) $\cdot$ Stefan Zimmermann $^{1}$ (D)
}

Received: 6 March 2020 / Revised: 6 March 2020 / Accepted: 22 April 2020 / Published online: 7 May 2020

(C) The Author(s) 2020

\begin{abstract}
The drift voltage required for operating ion mobility spectrometers implies high voltage isolation of either the ion source or the detector. Typically, the detector is grounded due to the sensitivity of the small ion currents to interferences and thus higher requirements for signal integrity than the ion source. However, for certain ion sources, such as non-radioactive electron emitters or electrospray ionization sources, or for coupling with other instruments, such as gas or liquid chromatographs, a grounded ion source is beneficial. In this paper, we present an isolated data acquisition interface using a 16 bit, 250 kilosamples per second analog to digital converter and fiber optic transmitters and receivers. All spectra recorded via this new data acquisition interface and with a grounded ion source show the same peak shapes and noise when compared with a grounded detector, allowing additional freedom in design.
\end{abstract}

Keywords Ion mobility spectrometer $\cdot$ Data acquisition $\cdot$ Amplifier $\cdot$ Isolated $\cdot$ Floating $\cdot$ Grounded ion source

\section{Introduction}

Ion mobility spectrometers (IMS) separate and characterize ions based on their motion under the influence of an electric field. Their possible analytical performance is directly related to the available drift voltage affecting both the resolving power and the signal-to-noise-ratio [1-5]. Typical drift voltages of ion mobility spectrometers are several kilovolts, requiring one end of the drift tube to be referenced to high voltage. Usually, the detector is at ground potential and the ion source is at high potential, as shown in Fig. 1, to avoid isolating the sensitive ion current signal.

However, for certain applications this is not the optimum configuration. For example, ionization sources requiring more complex driver electronics, such as non-radioactive electron emitters [6-8] or X-ray sources [9, 10]. Furthermore, other instruments such as gas chromatographs (GC) with heated transfer lines [11, 12], liquid chromatographs (LC) [13] or syringe pumps for direct electrospray ionization (ESI) [14,

Martin Lippmann

lippmann@geml.uni-hannover.de

1 Institute of Electrical Engineering and Measurement Technology, Department of Sensors and Measurement Technology, Leibniz University Hannover, Appelstr. 9A, 30167 Hannover, Germany
15] often require to be referenced to ground potential. Finally, a dual drift tube IMS for simultaneously recording both ion polarities $[16,17]$ requires at least one of the two detectors at high potential, as it is not possible to extract both the positive and negative ions from the ion source with two grounded detectors. Thus, in this paper we will discuss a simple isolated data acquisition interface, measuring the ion currents at high potential thus allowing to ground the ion source.

\section{Isolated data acquisition concept}

Figure 1 shows the basic setup of an IMS with a fieldswitching shutter, however, all explanations regarding the data acquisition also hold true for all other kinds of ion shutters and ion sources. In this basic setup the detector, amplifier, digitizer and data acquisition are grounded, while the ion source is at high potential defined by the drift voltage $\mathrm{U}_{\text {Drift }}$ which is applied to the drift rings through a resistor divider. Ions are generated between the injection electrode and the adjacent grid and are injected into the drift region by the pulse voltage $\mathrm{U}_{\text {Inj. }}$. Once injected the ions move towards the detector driven by the drift field and are finally detected with a faraday plate shielded by an aperture grid. The ion current is converted to a proportional voltage by a transimpedance amplifier and recorded by the data acquisition with an analog to digital 
Fig. 1 Schematic representation of an ion mobility spectrometer (IMS) in basic configuration with grounded detector and groundreferenced data acquisition. It is possible to either use an axial tritium source, an orthogonal X-ray source or an orthogonal ultraviolet (UV) source, which is in this configuration at high electric potential. The data acquisition consists of an analog to digital converter (ADC) connected to a microcontroller or a field programmable gate array (FPGA) via a serial peripheral interface (SPI), consisting of a conversion signal (Conv), a clock signal (SCLK) and a data signal (MISO/Data) from the ADC to the microcontroller or FPGA

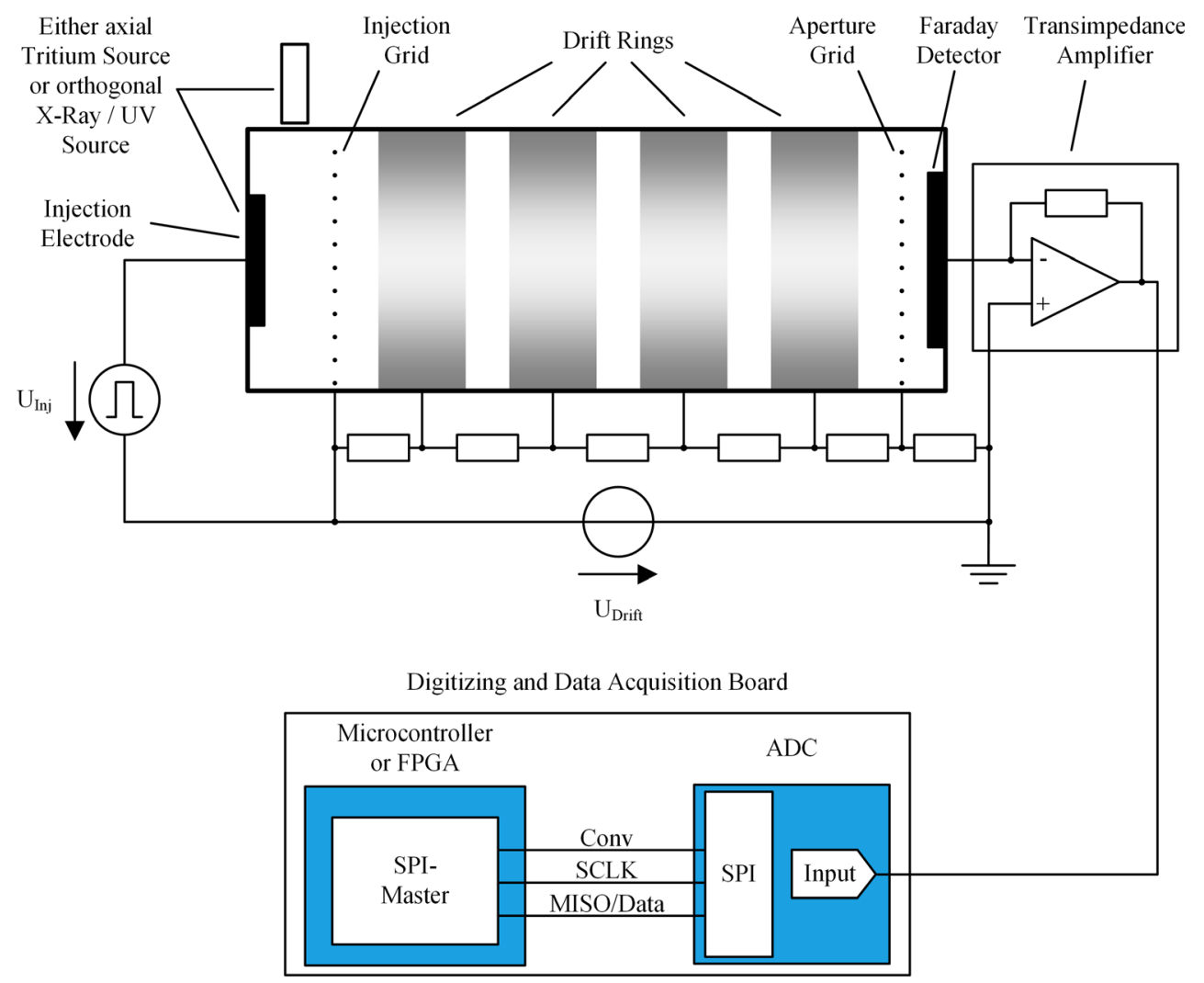

converter (ADC) connected to a digital data processing device, for example a microcontroller or field programmable gate array (FPGA). A microcontroller contains a processor combined with memories and additional specialized logics such as timers or interfaces for external components. Microcontrollers are versatile and easy to configure for most applications. An FPGA contains freely configurable logics that can be tailored specific applications. While requiring more effort to develop, they offer better performance for applications where many tasks have to be carried out at once, especially complicated timing.

The connection between the ADC and the microcontroller or FPGA typically requires a conversion signal (Conv) for determining the sampling point and a bus for data transfer, often the serial peripheral interface (SPI). While many different options exist, this is probably the most common one. The SPI interface consists of a clock signal (SCLK) and data signals in both directions. Here, only data transfer from the ADC to the microcontroller or FPGA is required. The line from the microcontroller or FPGA to the ADC is called master output, slave input (MOSI), the line from the ADC to the microcontroller or FPGA master input, slave output (MISO). The SCLK signal allows higher data rates than an unsynchronized transfer. With ADCs the MISO signal is often also called "Data" or "SDO".

For example, using an $\mathrm{ADC}$ with an acquisition rate of 250 kilosamples per second (ksps) requires $\mathrm{AD}$ conversion and data transmission to the microcontroller or FPGA within $4 \mu$ s per sample. Typically, AD conversion and data transmission cannot run simultaneously due to possible interference from the fast digital data signal on the sensitive analog signal. Therefore, if the $\mathrm{AD}$ conversion requires for example $3 \mu \mathrm{s}$, only $1 \mu$ s remains for data transmission. To transfer the data from a 16 bit $\mathrm{ADC}$ within $1 \mu$ s requires a transmission rate of at least 16 Mbit per second. Preventing any data corruption during transmission it is advised to transmit at a higher data rate, for example 20 Mbit per second. Using a data rate of 20 Mbit per second means that a single pulse on the data line is only 50 ns wide. Thus, sampling the data line is crucial. Therefore, the SPI interface uses synchronous sampling of data on SCLK edges with both devices acting on the same clock. In the SPI mode with clock polarity $(\mathrm{CPOL})=1$ and clock phase $(\mathrm{CPHA})=0$ shown in Fig. 2 , data is put onto the bus by the ADC on the falling edge of SCLK while the data is sampled by the microcontroller or FPGA on the rising edge of the SCLK as indicated by the green lines in Fig. 2.

In order to build an isolated data acquisition interface for IMS with a grounded ion source and floating detector as shown in Fig. 3, all these signals need to be isolated for example using an isolation chip or a fiber optics transmitter / receiver pair. At low data rates, this would not require any special effort. However, at high data rates required for transmitting the ADC data as stated above, the transmission delay of such optical isolators plays a significant role and requires a 
Conv.

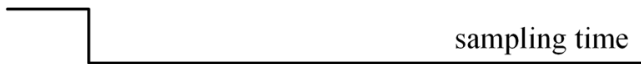
conversion time

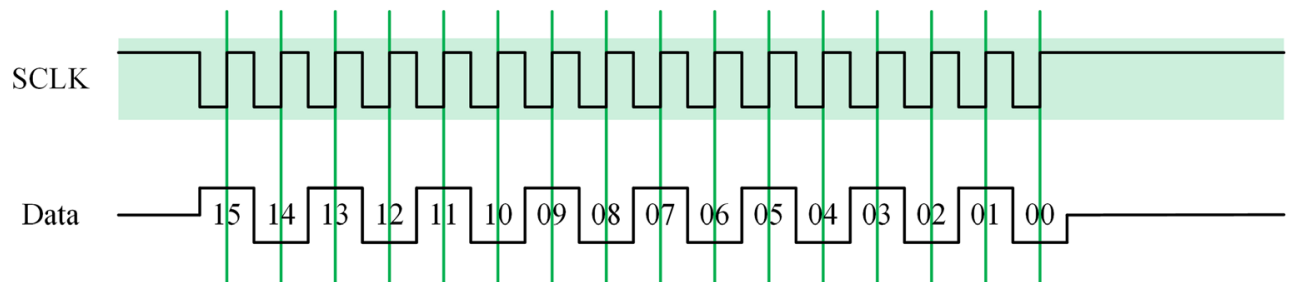

Fig. 2 Schematic timing diagram of a serial peripheral interface (SPI) data transmission with a 16 bit analog to digital converter (ADC). Data transmission begins with a falling edge of the conversion signal (Conv)

special design using four isolators [18-20]. While the conversion and data signals are simply isolated with a single isolator each, the SCLK signal is first sent to the ADC via one isolator and then sent back to the microcontroller or FPGA via another isolator, giving four isolators in total. subsequently data is put on the bus on the falling edge of the clock signal (SCLK) and is evaluated at the rising edge of SCLK, indicated by the green lines

The reason for this design is explained in Fig. 4, where all data transmitted from and to the microcontroller or FPGA are shown. The Conv and the SCLK signal are generated the same way as in Fig. 2 and sent to the ADC via the isolators. Upon receiving the SCLK signal change with a delay of one fiber

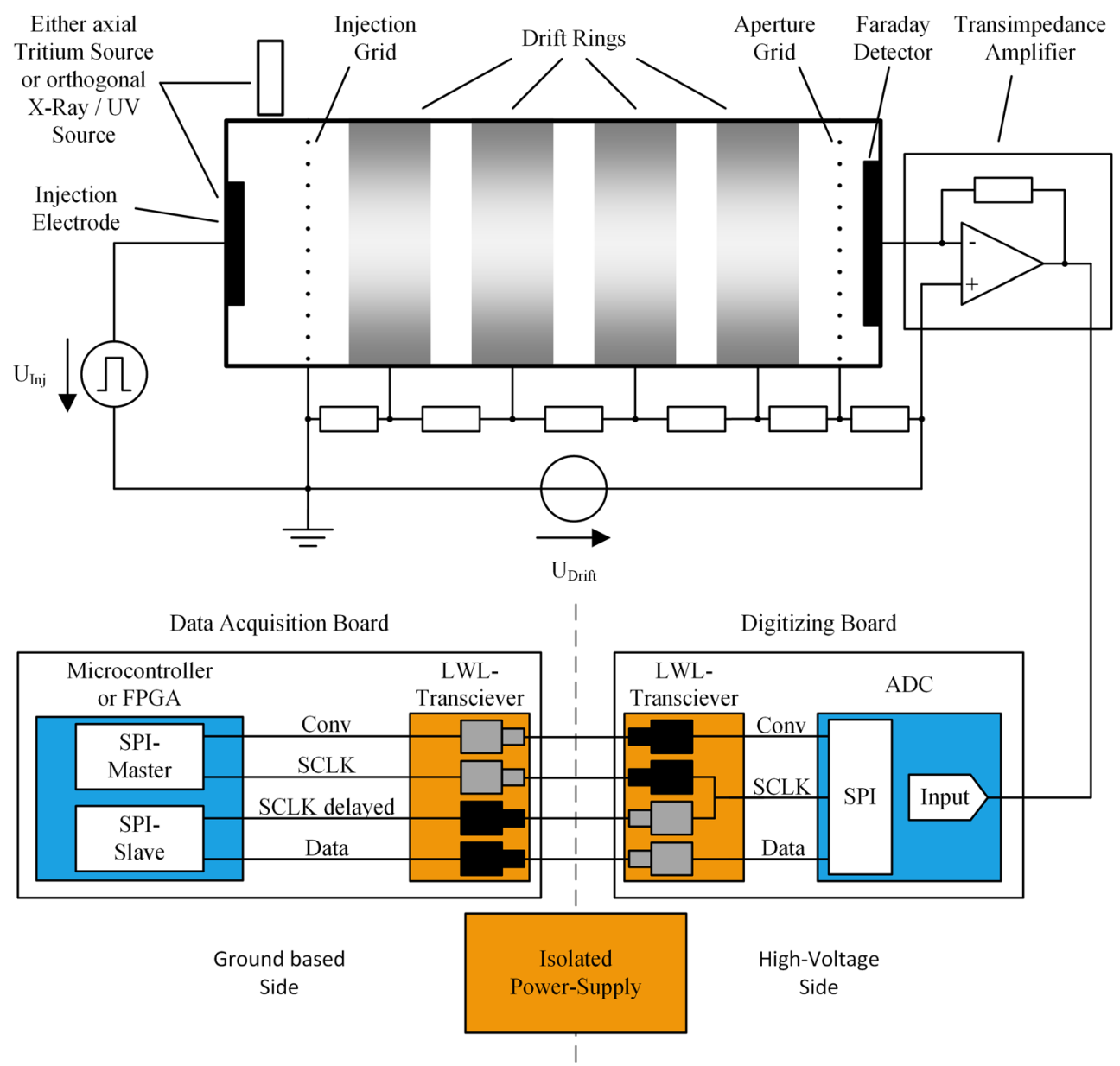

Fig. 3 Schematic representation of an ion mobility spectrometer (IMS) with detector at high electrical potential and an isolated data acquisition interface consisting of a data acquisition board at ground potential and a digitizing board at high potential powered by an isolated power supply. The ion source remains at ground potential and it is possible to use either an axial tritium source, an orthogonal X-ray source or an orthogonal ultraviolet (UV) source. For data acquisition the serial peripheral interface (SPI) between microcontroller or field programmable gate array (FPGA) and the analog to digital converter (ADC) consists of a SPI-master and a SPI-slave module implemented in the microcontroller or FPGA as well as a SPI interface in the ADC. They are connected through fiber optic transceivers (LWL-Transceiver). The SPI-Master generates the conversion signal (Conv) and the clock (SCLK), while the SPI-Slave receives the delayed clock (SCLK delayed) and the data from the ADC. Fiber optics transmitters are shown in grey and receivers in black 

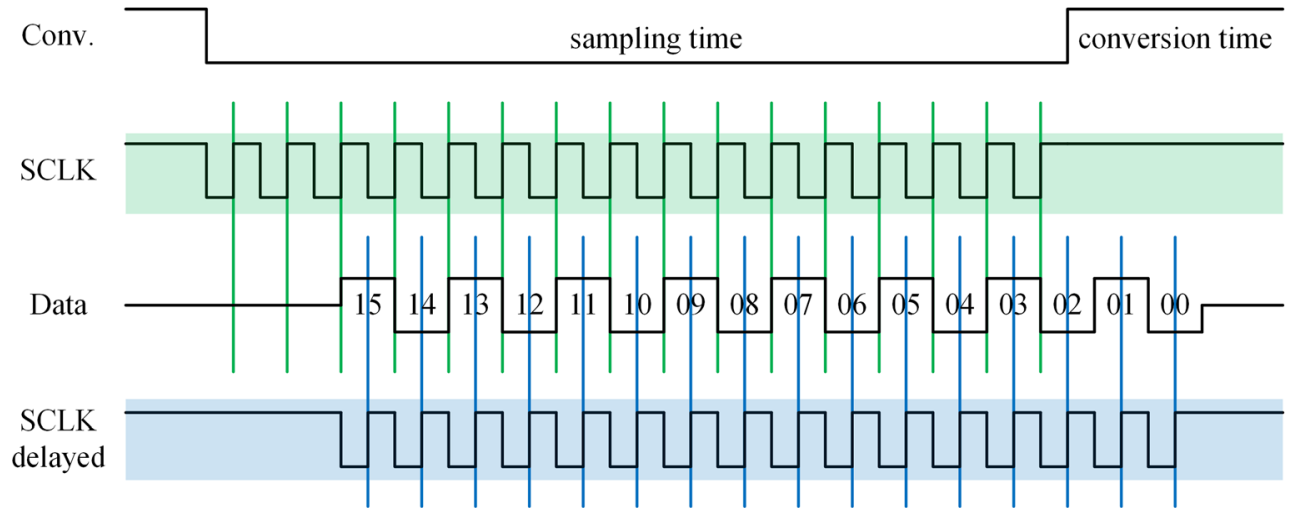

Fig. 4 Schematic timing diagram of a serial peripheral interface (SPI) data transmission through isolators. Data transmission begins with the falling edge of the conversion signal (Conv). The incoming data is significantly delayed in perspective of the clock signal (SCLK) and using the

optics transmission, the ADC outputs its data arriving at the microcontroller or FPGA with another delay of one transmission as shown in Fig. 4. Since each transmission of a signal over the isolation barrier consists of one fiber optic transmitter and one fiber optic receiver, the received data signal at the microcontroller or FPGA is delayed by four times the delay of a single transceiver compared to the generated SCLK. If for example the delay of a single transceiver is $30 \mathrm{~ns}$, the total delay is $120 \mathrm{~ns}$. As the SPI communication is clocked with $20 \mathrm{MHz}$, the data signal would arrive 2.4 periods of SCLK later at the microcontroller or FPGA. If data sampling is continued on the rising edges of SCLK, indicated by the green lines, the data become corrupted. To overcome this issue, the SCLK signal is also sent back from the isolated side and used to sample the data. Now both the SCLK and data undergo the same delay and the data can be evaluated correctly using SCLK delayed as indicated by the blue lines. If the delay is known, it is also possible to simplify the setup by directly generating a delayed SCLK on the digital processing device [21].

In this case, the design requires two SPI interfaces implemented at the microcontroller or FPGA on the data acquisition board, one as a master for generating the SCLK signal and one as a slave for receiving the data synchronized to the delayed SCLK. However, even most low cost microcontrollers are equipped with two SPI interfaces, such as ATmega328P used on the Arduino Uno. Furthermore, if the microcontroller just sends the SCLK signal and no additional data to the ADC, it would be also possible to use a simple timer to generate a $50 \%$ duty cycle signal with the desired frequency. In this case, only a single SPI interface is required.

\section{Experimental and electronics}

A data acquisition system consisting of two different printed circuit boards (PCBs) using the scheme shown in Fig. 3 was normal sampling time (green) does not yield valid data. Only when using an equally delayed SCLK and sampling at these times (blue), the signal is valid again

designed and manufactured at our institute and is displayed in Fig. 5.

The digitizing board consists of an ADC from Linear Technology (LTC1864) with 16 bit resolution and a sampling rate of $250 \mathrm{ksps}$ and four fiber optic transceivers from Broadcom (AFBR-1624Z and AFBR-2624Z), providing data rates of up to $60 \mathrm{Mbit}$ per second fast enough for used data rates of 20 Mbit per second.

The data acquisition board contains an FPGA from Xilinx (Spartan-3E) and a microcontroller from Microchip
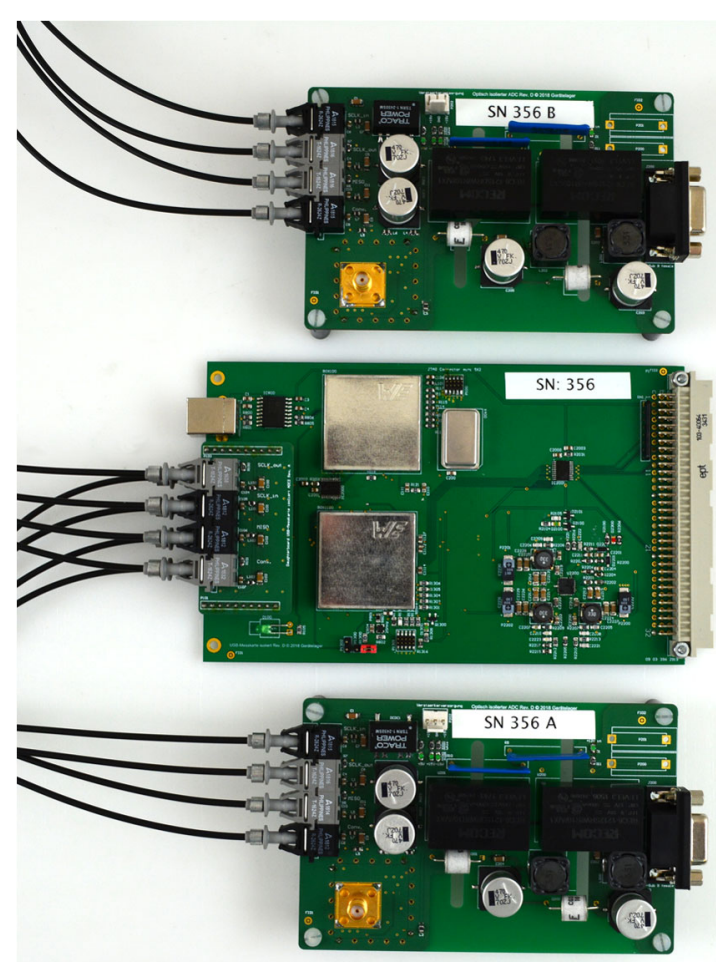

Fig. 5 Photo of the data acquisition interface with two digitizing boards at the top and the bottom connected to the data acquisition board in the middle via fiber optics 
(AT90USB1287), which are used to communicate with the ADC and provide a computer interface as well as also two times four fiber optic transceivers. The FPGA provides four SPI interfaces in order to simultaneously use two digitizing boards for a dual drift tube IMS. Furthermore, the FPGA can also be used to provide additional timing functions, e.g. additional pulses for a FAT-IMS [22, 23].

For the isolated power supply, two isolated RECOM DC/ DC-converters in series (RECOM 1215SRW/R10 and RECOM 1215DRW/R10) are used to provide an isolation of up to $12 \mathrm{kV}$. For lower isolation, only one would be sufficient.

For first experiments we used our standard drift tube IMS with a field switching shutter [24] operated at an injection voltage of $500 \mathrm{~V}$, a drift voltage of $5 \mathrm{kV}$ and with $77 \mathrm{~mm}$ drift length $[3,25]$. Ions were generated using a tritium ionization source. For current amplification our fast, low-noise amplifier was employed [26]. The amplifier is also powered by the isolated power supply of the data acquisition board. The IMS was operated at $40{ }^{\circ} \mathrm{C}$ and at 1013 mbar using purified air with a dew point of $-90^{\circ} \mathrm{C}$ as drift and sample gas.

Furthermore, an in-house developed isolated $5 \mathrm{kV}$ power supply was used for the drift voltage, allowing us to change between the setups in Figs. 1 and 3 by simply moving the ground lead between ion source and detector. Thus, we can directly compare the performance of both configurations.

\section{Results and discussion}

Figure 6 shows two ion mobility spectra, one measured with grounded ion source and the other measured with grounded detector in combination with the new data acquisition board. Peak position, peak shape and baseline are indistinguishable.

Figure 7 shows the standard deviation of the baseline, which characterizes the noise as a function of different averages using a grounded ion source and a grounded detector. Furthermore, a fit is shown, indicating that the noise drops with the square root of the number of averages as expected for white noise. The standard deviation was calculated for both configurations in the time period between $9 \mathrm{~ms}$ and $13 \mathrm{~ms}$, where no peaks were present in the spectra. There is no difference between both configurations, except minor differences for small numbers of averages. Thus, it is possible to operate the detector at any desired potential using our new data acquisition interface.

\section{Conclusion}

In this paper, we presented a newly developed isolated data acquisition interface for IMS with grounded ion sources that preserves the quality of the detector signal compared to the standard setup with grounded detector. The analog signal is

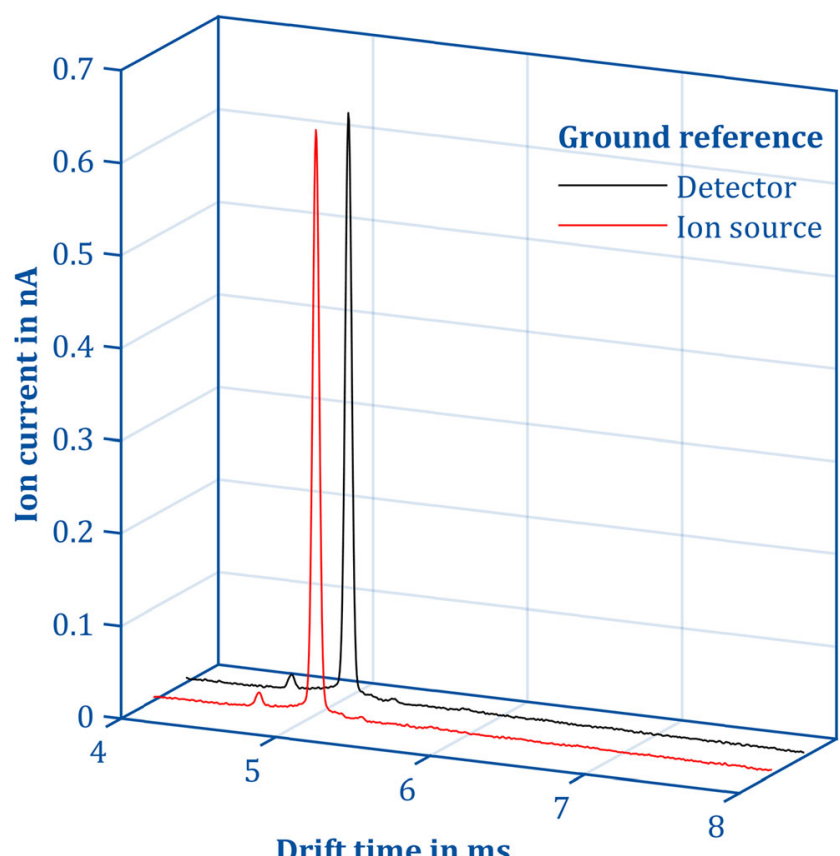

Fig. 6 Ion mobility spectra acquired using a grounded detector (black) and a grounded ion source (red)

directly digitized on high electric potential before transmitting it to ground potential via fiber optics, thus preventing additional noise or signal distortion. In the current setup, the isolated data acquisition is based on a 16 bit $\mathrm{ADC}$ with a maximum data acquisition rate of $250 \mathrm{ksps}$ and an isolated power supply with an isolation of $12 \mathrm{kV}$ maximum working voltage. Using this setup, it is now possible to place the ground

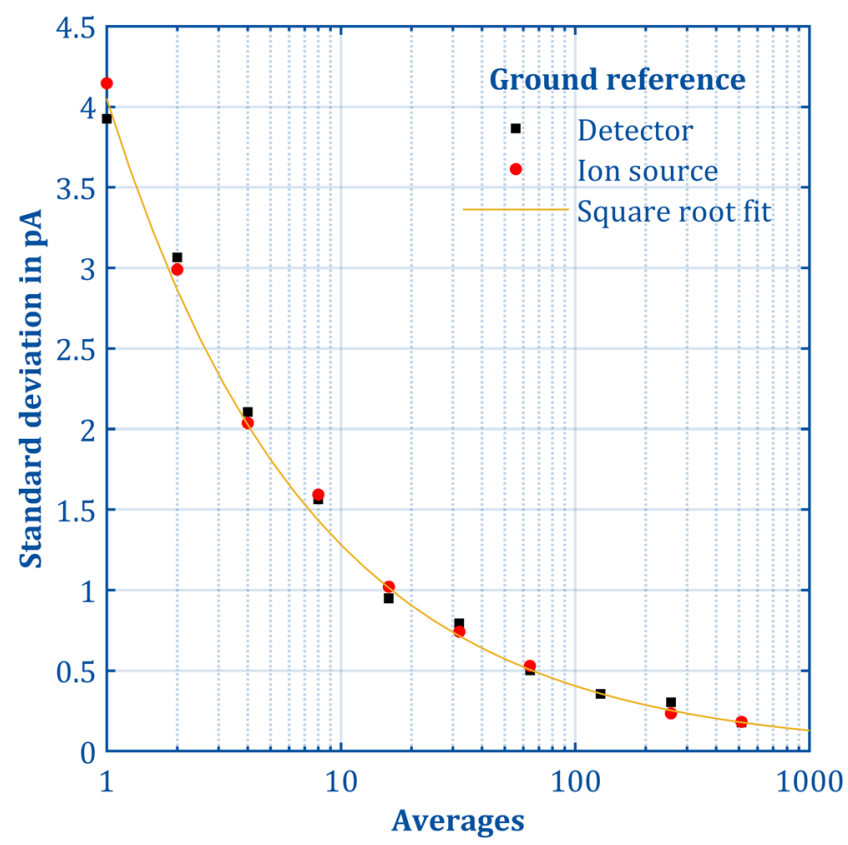

Fig. 7 Standard deviation of the baseline as a function of the averages using a grounded detector (black) and a grounded ion source (red) 
potential at any desired point of the drift tube, allowing additional design freedom.

Funding information Open Access funding provided by Projekt DEAL. Funded by the Deutsche Forschungsgemeinschaft (DFG, German Research Foundation) - 396430937.

\section{Compliance with ethical standards}

Conflict of interest The authors declare that they have no conflict of interest.

Open Access This article is licensed under a Creative Commons Attribution 4.0 International License, which permits use, sharing, adaptation, distribution and reproduction in any medium or format, as long as you give appropriate credit to the original author(s) and the source, provide a link to the Creative Commons licence, and indicate if changes were made. The images or other third party material in this article are included in the article's Creative Commons licence, unless indicated otherwise in a credit line to the material. If material is not included in the article's Creative Commons licence and your intended use is not permitted by statutory regulation or exceeds the permitted use, you will need to obtain permission directly from the copyright holder. To view a copy of this licence, visit http://creativecommons.org/licenses/by/4.0/.

\section{References}

1. Siems WF, Wu C, Tarver EE, Hill HH Jr, Larsen PR, McMinn DG (1994) Measuring the resolving power of ion mobility spectrometers. Anal Chem 66(23):4195-4201

2. Kanu AB, Gribb MM, Hill HH (2008) Predicting optimal resolving power for ambient pressure ion mobility spectrometry. Anal Chem 80(17):6610-6619

3. Kirk AT, Zimmermann S (2015) Pushing a compact $15 \mathrm{~cm}$ long ultra-high resolution drift tube ion mobility spectrometer with $\mathrm{R}=$ 250 to $R=425$ using peak deconvolution. Int J Ion Mobil Spec 18(1-2):17-22

4. Kirk AT, Zimmermann S (2015) An analytical model for the optimum drift voltage of drift tube ion mobility spectrometers with respect to resolving power and detection limits. Int $\mathrm{J}$ Ion Mobil Spec 18(3-4):129-135

5. Kirk AT, Bakes K, Zimmermann S (2017) A universal relationship between optimum drift voltage and resolving power. Int J Ion Mobil Spec 20(3-4):105-109

6. Gunzer F, Ulrich A, Baether W (2010) A novel non-radioactive electron source for ion mobility spectrometry. Int J Ion Mobil Spec 13(1):9-16

7. Cochems P, Runge M, Zimmermann S (2014) A current controlled miniaturized non-radioactive electron emitter for atmospheric pressure chemical ionization based on thermionic emission. Sensors Actuators A Phys 206:165-170

8. Bunert E, Berger M, Kirk AT, Zimmermann S (2019) Nonradioactive electron source with nanosecond pulse modulation for atmospheric pressure chemical ionization. Rev Sci Instrum 90(11): 113306

9. Reinecke T, Kirk AT, Heptner A, Niebuhr D, Bottger S, Zimmermann S (2016) A compact high-resolution X-ray ion mobility spectrometer. Rev Sci Instrum 87(5):53120

10. Bunert E, Reinecke T, Kirk AT, Bohnhorst A, Zimmermann S (2018) Ion mobility spectrometer with orthogonal X-ray source for increased sensitivity. Talanta 185:537-541

11. St. Louis RH, Siems WF, Hill HH (1989) Evaluation of direct axial sample introduction for ion mobility detection after capillary gas chromatography. J Chrom A 479:221-231

12. Kanu AB, Hill HH (2008) Ion mobility spectrometry detection for gas chromatography. J Chrom A 1177(1):12-27

13. Piendl SK, Raddatz C-R, Hartner NT, Thoben C, Warias R, Zimmermann S, Belder D (2019) 2D in seconds: coupling of Chip-HPLC with ion mobility spectrometry. Anal Chem 91(12): 7613-7620

14. Chen C, Hou K, Wang W, Li J, Li H (2014) Ambient temperature nanoelectrospray ion mobility detector for high performance liquid chromatography in determining amines. J Chrom A 1358:192-198

15. Reinecke T, Kirk AT, Ahrens A, Raddatz C-R, Thoben C, Zimmermann S (2016) A compact high resolution electrospray ionization ion mobility spectrometer. Talanta 150:1-6

16. Spangler GE, Wroten JF (1984) Apparatus for simultaneous detection of positive and negative ions in ion mobility spectrometry US4445038

17. Vautz WD (2010) Ionenbeweglichkeitsspektrometer. DE102009008266

18. Kugelstadt T (2011) Extending the SPI bus for long-distance communication. Anal Appl J

19. Cantrell MS, Goswami B (2014) MS-2689: isolating SPI for high bandwidth sensors. Analog Devices Technical Article

20. Jackson M, Long S (2017) Add robust and reliable isolation to your high speed SPI communications. Maxim Integrated Technical Article

21. Goswami B, Cantrell MS, Chen B Integrated delayed clock for high speed isolated SPI communication. US8928383B2

22. Bohnhorst A, Kirk AT, Berger M, Zimmermann S (2018) Fast orthogonal separation by superposition of time of flight and field asymmetric ion mobility spectrometry. Anal Chem 90(2):11141121

23. Bohnhorst A, Kirk AT, Yin Y, Zimmermann S (2019) Ion fragmentation and filtering by alpha function in ion mobility spectrometry for improved compound differentiation. Anal Chem 91(14):89418947

24. Kirk AT, Zimmermann S (2014) Bradbury-Nielsen vs. Field switching shutters for high resolution drift tube ion mobility spectrometers. Int J Ion Mobil Spec 17:131-137

25. Kirk AT, Allers M, Cochems P, Langejürgen J, Zimmermann S (2013) A compact high resolution ion mobility spectrometer for fast trace gas analysis. Analyst 138(18):5200-5207

26. Cochems P, Kirk AT, Zimmermann S (2014) In-circuitmeasurement of parasitic elements in high gain high bandwidth low noise transimpedance amplifiers. Rev Sci Instrum 85(12): 124703

Publisher's note Springer Nature remains neutral with regard to jurisdictional claims in published maps and institutional affiliations. 